\title{
Modellierung des Einflusses von Wildverbiss auf die Schutzwaldentwicklung an der Rigi-Nordlehne
}

\author{
Golo Stadelmann \\ Monika Frehner \\ Andri Baltensweiler \\ Harald Bugmann
}

\author{
Waldökologie, Institut für Terrestrische Ökosysteme, ETH Zürich $(\mathrm{CH})$ * \\ Waldbau und Waldmanagement, Institut für Terrestrische Ökosysteme, ETH Zürich (CH) \\ Landressourcenbeurteilung, Eidgenössische Forschungsanstalt für Wald, Schnee und Landschaft (CH) \\ Waldökologie, Institut für Terrestrische Ökosysteme, ETH Zürich (CH)
}

\author{
Modelling the impact of ungulate browsing on the development \\ of the protective forests of the Rigi-Nordlehne
}

\begin{abstract}
Forests on the northern slopes of the Rigi Mountain in central Switzerland ("Rigi-Nordlehne") have a key protective function against rockfall, erosion, landslides, flooding and debris flows. Forest management endeavours to guarantee that these forests can maintain their role in the long term. Thus multi-story canopies are favored, being resistant and resilient to natural disturbances such as windthrow. In addition, the mixture of tree species should conform to the guidelines of the national project "Sustainability and success monitoring in protection forests" (NaiS). However, at the present time an imbalance in tree species is evident in the regeneration layer, due to the considerable impact of browsing by wild ungulate populations in these beech and fir forests. To predict future mixtures of tree species, the spatially explicit model of forest development Rigfor was developed and applied to simulate and compare two scenarios of forest dynamics. The scenario "current browsing" suggests that silver fir cannot regenerate and that regeneration of maple and beech is greatly hindered. In the scenario "reduced browsing", however, a species mixture conforming to the national guidelines can be achieved in the regeneration layer. The simulations show that the amount of beech timber decreases whichever the scenario, while for the larger fir trees in diameter classes above $35 \mathrm{~cm}$ no changes are to be expected in the coming decades. However, from 2082 onwards a continuing high browsing rate would lead to the elimination of nearly all silver fir trees at the pole stage and in the long run silver fir may be completely eradicated from these stands. We conclude that under a dense canopy of old trees, silver fir and beech can be regenerated if ungulate browsing is considerably reduced. Spruce regeneration however requires more light and this is not often found on the northern slopes of the Rigi Mountain. Opening up the canopy to foster regeneration of the desired mixture of species is a key measure in order to maintain the long-term protective function of these forests. This will only be possible, however, if the current high browsing intensity can be reduced.
\end{abstract}

Keywords: succession, forest dynamics, rockfall, protection forest, natural hazards doi: $10.3188 /$ szf. 2011.0355

*Universitätstrasse 16, CH-8092 Zürich, E-Mail golo.stadelmann@env.ethz.ch

$\mathrm{D}$ ie Wälder an der Rigi-Nordlehne leisten wirksamen Schutz vor Stein- und Blockschlag, Erosion, Rutschungen, Hochwasser und Murgang. In höheren Lagen verhindern sie zudem das Anreissen von Lawinen. Aufgrund dieser Gefahrenprozesse ist es essenziell, dass der Wald in einen möglichst störungsresistenten Zustand gebracht wird und dieser Zustand so gut wie möglich erhalten wird. Störungsereignisse wie zum Beispiel Wind, Hagelstürme, Schneedruck oder Borkenkäferbefall beeinträchtigen die Schutzwirksamkeit eines Waldes. Die Schutzwaldbewirtschaftung stellt deshalb eine grosse Herausforderung dar. Weil damit gerechnet werden muss, dass Störungsereignisse in Zukunft vermehrt auftreten (North et al 2007), wird diese Aufgabe noch anspruchsvoller werden. Durch die Schutzwaldbewirtschaftung will man die Störungsresistenz der Bestände erhöhen. Davis \& Moritz (2001) definieren Resistenz als die Fähigkeit eines Systems, trotz Störung im selben Zustand zu verweilen. Des Weiteren soll nach dem Eintreten eines Störungsereignisses die Zeitdauer mit beeinträchtigter Schutzwirkung des Bestandes möglichst kurz gehalten werden, die Resilienz des Bestandes soll also möglichst hoch sein. Resilienz gilt als die Fähigkeit und Geschwindigkeit des Systems, sich nach einer Störung wieder in den ursprünglichen Zustand zurückzuentwickeln (Davis \& Moritz 2001). Gemäss der Wegleitung «Nachhaltigkeit und Erfolgskontrolle im Schutzwald» (NaiS; Frehner et al 2005) kann eine 
Abb 1 Blick auf die Rigi-Nordlehne.

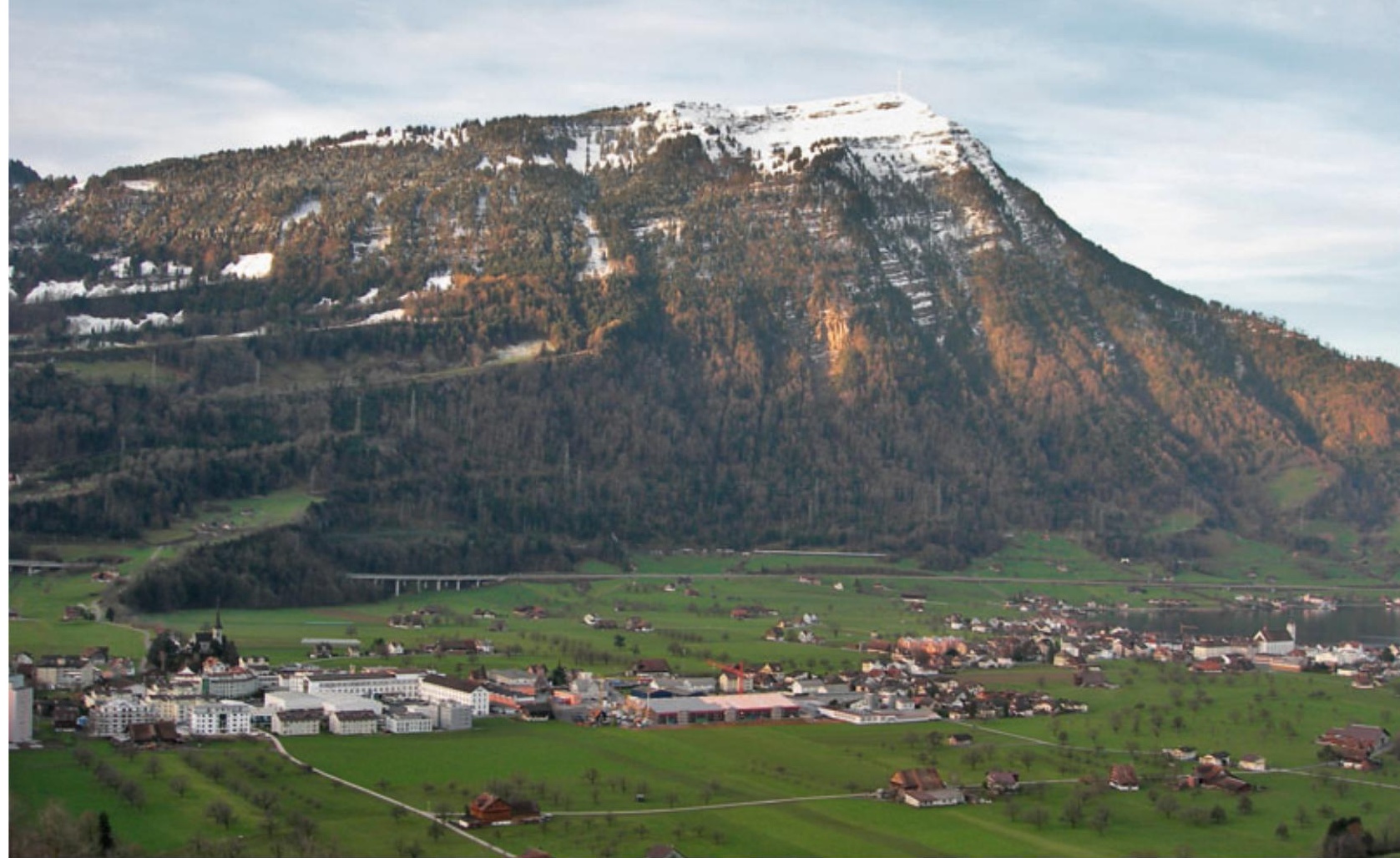

dauerhafte Schutzwirkung erzielt werden, indem ein mehrschichtiger Bestandesaufbau gefördert wird, der über eine hohe Störungsresistenz und Resilienz verfügt.

Ein mehrschichtiger Bestandesaufbau führt dazu, dass verschiedene Alters- beziehungsweise Durchmesserklassen neben- und übereinander vorkommen. Durch Störungen entstehen daher oft nur kleine Bestandesöffnungen, in denen sich relativ rasch Verjüngung einstellen kann. An der RigiNordlehne ist grundsätzlich genügend Ansamung vorhanden. Im Tannen-Buchen-Wald führt starker Wildverbiss jedoch dazu, dass die Ansamung nicht aufwachsen kann (Frehner \& Schwitter 2008). Es ist nicht einfach, die langfristigen Konsequenzen einer bestimmten Verbissbelastung für die Walddynamik zu bestimmen. Anhand von Simulationsmodellen kann aber abgeschätzt werden, welchen Einfluss Veränderungen in der Verjüngung auf die Walddynamik haben. Um die zukünftige Schutzwirkung eines Waldes abschätzen zu können, muss eine räumlich explizite Bestandesentwicklung simuliert werden, da die Schutzwirkung nur über grössere Flächen bestimmt werden kann und sich Topografie und Waldstruktur im Gebirge oft auf kleinem Raum ändern. Daher konnte in dieser Arbeit nicht auf bestehende Modelle wie das Schutzwaldmodell (Brang et al, 2004) oder ForClim (Bugmann 1999) zurückgegriffen werden.

Ziel dieser Arbeit war es, die zukünftige Walddynamik an der Rigi-Nordlehne abzuschätzen. Dazu wurde das räumlich explizite Waldmodell «Rigfor» entwickelt, das im Folgenden vorgestellt wird. Si- mulationen der Waldentwicklung über 100 Jahre ermöglichen es, die zwei Entwicklungsszenarien «aktueller Wildverbiss» und «reduzierter Wildverbiss» miteinander zu vergleichen. Dabei wird insbesondere auf die Entwicklung der Baumartenzusammensetzung und deren Konsequenzen für die Stabilität und die Schutzwirkung der Bestände eingegangen.

\section{Material und Methoden}

\section{Untersuchungsgebiet}

Die Rigi-Nordlehne (Abbildung 1; siehe auch Karte in Gasser et al 2011, dieses Heft) befindet sich in der Standortregion Nördliche Randalpen. Kühle Temperaturen und mässige tägliche und jahreszeitliche Temperaturschwankungen sowie hohe Niederschläge von über $1200 \mathrm{~mm}$ pro Jahr auf $1000 \mathrm{~m}$ ü. M. charakterisieren diesen Klimatyp (Frehner et al 2005). Die Horizontaldistanz zwischen Rigi Kulm (1797.5 m ü. M.) und dem Zugersee (413 m ü. M.) beträgt lediglich rund zwei Kilometer. Entsprechend weist die Rigi-Nordlehne ein hohes Gefälle auf. Die Waldfläche an der Rigi-Nordlehne beträgt rund 460 Hektaren, wovon 270 Hektaren der Buchenwaldstufe und 190 Hektaren der Tannen-Buchen-Waldstufe zugehören (Hug 2007). In der Buchenwaldstufe, die bis auf eine Höhe von rund 850-900 m ü. M. steigt, dominieren wüchsige Buchenwälder mit mittleren Bedingungen. Mit zunehmender Höhe über Meer steigen die Nadelholzanteile an, wobei diese im Naturwald hauptsächlich aus Tanne bestehen würden. Natürlicherweise würden Tanne und Bu- 
che diese Wälder dominieren. Bergahorn, Fichte und Esche können ebenfalls beigemischt sein. Im Idealfall trägt die Fichte maximal 20\% zur Stammzahl bei (Frehner et al 2005).

Die heutige Stammzahlverteilung in den Tannen-Buchen-Wäldern der Rigi-Nordlehne entspricht bei Weitem nicht diesem Ideal. Selbst die minimalen Anforderungen gemäss NaiS können in mehr als 40\% der Tannen-Buchen-Wälder nicht erfüllt werden. Im Baumholz ist die Tanne genügend, die Buche hingegen eindeutig zu wenig vertreten. Nur etwa die Hälfte der Flächen im Tannen-Buchen-Wald weist einen ausreichenden Buchenanteil auf. Anders sieht es mit den Fichtenanteilen aus, welche heute oft $\mathrm{zu}$ hoch sind (Frehner \& Schwitter 2008). Die Mischung der Jungwaldflächen entspricht ebenfalls nicht dem minimalen Anforderungsprofil. Die tiefen Buchenanteile sind aufgrund der Zusammensetzung im Baumholz zu erwarten; anders ist es aber bei den Tannenanteilen: Obwohl genügend Samenbäume vorhanden sind und die Ansamung gut ist, ist die Tanne im Jungwald nicht vertreten. Dies zeigt, dass bei der Tannenverjüngung schon seit langer Zeit Probleme bestehen. Diese werden auf den hohen Schalenwildbestand zurückgeführt (Frehner \& Schwitter 2008).

\section{Waldentwicklungsmodell}

Das räumlich explizite Modell Rigfor (Stadelmann 2008) ist ein rasterbasiertes Modell mit einer Zellengrösse von einer Are. Einerseits ist so jede Zelle genügend gross, damit ein Gerüstbaum darin Raum findet und Ansamung möglich ist. Andererseits ist die Zelle wesentlich kleiner als typische Bestände, was eine Simulation der Bestandesdynamik ermöglicht. Weil das Modell auf empirischen Regeln basiert, kann Rigfor als regelbasiertes Modell bezeichnet werden. Bei der Definition der Regeln wurde berücksichtigt, dass der Wald im Untersuchungsgebiet bewirtschaftet wird. Es wurden also nicht nur ökologische Kriterien, sondern auch Bewirtschaftungsregeln implementiert, die den Anforderungen des Schutzwaldmanagements entsprechen. Die Software ArcGIS Desktop 9.2 der Firma ESRI bildete die Grundlage für die Implementation des Waldmodells, welches mit Python programmiert wurde.

\begin{tabular}{|l|c|c|c|}
\hline Baumarten & $\begin{array}{c}\text { Lebensdauer im } \\
\text { Urwald }\end{array}$ & $\begin{array}{c}\text { Produktionszeitraum } \\
\text { im Wirtschaftswald } \\
\text { des Mittellands }\end{array}$ & $\begin{array}{c}\text { Produktionszeitraum } \\
\text { im Wirtschaftswald } \\
\text { der Voralpen }\end{array}$ \\
\hline Fichte & $300-350$ & 110 & $140-150$ \\
\hline Tanne & $350-460$ & 120 & $130-140$ \\
\hline Buche & $230-260$ & 120 & $150-160$ \\
\hline
\end{tabular}

Tab 1 Lebensdauer von Buche, Tanne und Fichte in Urwäldern Osteuropas (Korpel 1995) und Produktionszeiträume im Wirtschaftswald (Schütz 2002) in Jahren. Die angegebene Lebensdauer der Fichte im Urwald entspricht derjenigen in ihrem optimalen Verbreitungsgebiet. Für natürliche Fichtenwälder der Schweiz wird ein Baumalter von 250 bis 300 Jahren angenommen.

\section{Modellannahmen}

Weil die Walddynamik eines realen Waldes simuliert werden sollte, mussten die Systemzustände des Modells so definiert werden, dass der Anfangszustand der Simulationen aus der Bestandeskarte 2002 abgeleitet werden konnte. Die Bestandeskarte enthält Angaben zu Entwicklungsstufe, Deckungsgrad, Nadelholz- und Laubholzanteil sowie den Anteilen von Fichte, Tanne, Buche, übrigem Laubholz und übrigem Nadelholz. Um diese Angaben im Modell zu implementieren, wurden die folgenden vier Annahmen getroffen:

1) Entwicklungsstufe: Alle Bäume eines einschichtigen Bestandes wurden derselben Entwicklungsstufe zugeordnet. Bei stufigen und zweischichtigen Beständen wurden die Zellen in Zellen mit unterschiedlichen Systemzuständen umgerechnet.

2) Deckungsgrad: In der Bestandeskarte von 2002 wurde der Deckungsgrad in den Klassen < 20\%, 20-60\%, 60-90\% und > 90\% angegeben. Wenn der Deckungsgrad unter $100 \%$ liegt, so beträgt der Lückenanteil = 1 - Deckungsgrad. Es wird zudem angenommen, dass der Deckungsgrad aller Bestände sich in der Klassenmitte befindet (also bei 10, 40, 75 respektive 95\%).

3) Baumartenmischung: Im Waldmodell wird analog zur Bestandeskarte zwischen Fichte (FI), Tanne (TA), Buche (BU) und übrigem Laubholz (UL) unterschieden. Übriges Laubholz besteht im Wesentlichen aus Ahorn und Esche. Da der Anteil an übrigem Nadelholz an der Rigi-Nordlehne als unter 5\% liegend kartiert wurde, wurde er im Modell vernachlässigt.

4) Lebenszyklus: Die Verweilzeiten der Baumarten in den Systemzuständen des Waldmodells wurden anhand von Literaturangaben für den Buchenwald und den Tannen-Buchen-Wald (Tabellen 1 und 2) festgelegt. Gegenüber der Bestandeskarte wurde der Zustand Lücke (LU) ergänzt. Es wurde angenommen, dass übriges Laubholz genauso schnell wächst wie die Fichte, jedoch eine längere Lebensdauer hat. Für den Buchenwald beträgt das maximale Alter 140 Jahre, im Tannen-Buchen-Wald dauert der Zyklus 180 Jahre.

Zur Simulation der Verjüngung und der Störungen wurden drei weitere Annahmen hinzugefügt: 5) Baumartenmischung: Alle Zellen, die sich in den Zuständen Baumholz I-III befinden und nicht weiter als $40 \mathrm{~m}$ von der zu verjüngenden Lücke entfernt wachsen, tragen zur Baumartenmischung bei. 6) Störungen: Zellen, die das Baumholz noch nicht erreicht haben, werden durch Störungen nicht in den Lückenzustand zurückgeworfen. Es wird angenommen, dass im Falle einer Störung unterdrückte Individuen freigestellt werden und daher überleben können. Störungen wirken erst ab dem Baumholz. Die Sturmanfälligkeit der vertretenen Baumarten ist in absteigender Reihenfolge Fichte $>$ Tanne $>$ Buche $>$ übriges Laubholz (Schütz 2002). 


\begin{tabular}{|c|c|c|c|c|c|c|c|}
\hline \multirow{2}{*}{$\begin{array}{l}\text { Höhenstufe } \\
\text { und Baumart }\end{array}$} & \multirow{2}{*}{$\begin{array}{c}\text { Lebensalter } \\
\text { (J) }\end{array}$} & \multirow{2}{*}{$\begin{array}{l}\text { Lücke } \\
\text { (LU) }\end{array}$} & \multicolumn{5}{|c|}{ Maximaler Indexwert im Zustand } \\
\hline & & & $\begin{array}{l}\text { Jungwald } \\
\text { (JW) }\end{array}$ & $\begin{array}{c}\text { Stangenholz } \\
\text { (STH) }\end{array}$ & $\begin{array}{c}\text { Baumholz I } \\
\text { (B I) }\end{array}$ & $\begin{array}{c}\text { Baumholz II } \\
\text { (B II) }\end{array}$ & $\begin{array}{c}\text { Baumholz III } \\
\text { (B III) }\end{array}$ \\
\hline \multicolumn{8}{|l|}{ Buchenwald } \\
\hline Fichte & 120 & 1 & 6 & 9 & 12 & 15 & 24 \\
\hline Tanne & 200 & 1 & 9 & 13 & 16 & 23 & 40 \\
\hline Buche & 160 & 1 & 7 & 11 & 15 & 19 & 32 \\
\hline \multicolumn{8}{|c|}{ Tannen-Buchen-Wald } \\
\hline Fichte & 180 & 1 & 8 & 13 & 17 & 21 & 36 \\
\hline Tanne & 300 & 1 & 15 & 19 & 23 & 31 & 60 \\
\hline Buche & 200 & 1 & 11 & 14 & 21 & 27 & 40 \\
\hline
\end{tabular}

Tab 2 Systemzustände im Buchen- und Tannen-Buchen-Wald. Für jeden Systemzustand wurde in Abhängigkeit der Baumart ein Indexwert festgelegt, bei welchem die Zelle in einen nächsten Zustand wechselt. Das Baumalter entspricht dem Indexwert multipliziert mit fünf Jahren.

7) Die Wachstumsgeschwindigkeit der Bäume und die Störungsraten bleiben über den simulierten Zeitraum konstant.

\section{Modellstruktur}

Rigfor simuliert die Waldentwicklung in Zeitschritten von fünf Jahren. Dies bedeutet, dass die gesamte Entwicklung des Ökosystems und alle Störungen innerhalb eines Zeitschritts zu einem diskreten Ereignis zusammengefasst werden. Während jedes Zeitschritts werden die vier Prozessgruppen Wachstum und Mortalität, Regeneration, Baumartendifferenzierung und Störungsregime in der genannten Reihenfolge aufgerufen.

In der Prozessgruppe Wachstum und Mortalität wird die Bestandesentwicklung simuliert, wobei alle Individuen einer Art gleich schnell wachsen und die gleiche Lebensdauer haben (Tabelle 1). Eine Zelle befindet sich jeweils in genau einem Systemzustand (Tabelle 2), wobei die Reihenfolge der Entwicklung vorgeschrieben ist: Lücke (LU) > Jungwald (JW) > Stangenholz (STH) $>$ Baumholz I (B I) $>$ Baumholz II (B II) > Baumholz III (B III). Eine rückschreitende Entwicklung ist nicht möglich. Lücken können entweder durch altersbedingtes Absterben beziehungsweise Holzschlag (B III > LU) oder durch Störungen (B I - B III > LU) entstehen. So wechselt beispielsweise eine Fichtenzelle im Buchenwald nach 120 Jahren (24 Zeitschritte à fünf Jahre) vom B III zu Lücke.

Die Prozessgruppe Regeneration prüft die Verfügbarkeit von Samenbäumen. Die Baumartenanteile der Verjüngung entsprechen der prozentualen Artenmischung der Samenbäume. Wenn im Radius von 40 m keine Samenbäume vorhanden sind, kann die Zelle den Systemzustand nicht wechseln, d.h., sie verbleibt im Zustand LU.

Wenn auf einer Jungwaldzelle mehrere Baumarten vorhanden sind, so findet beim Übergang ins Stangenholz eine Baumartendifferenzierung statt. Dabei kann sich nur eine Baumart durchsetzen. Sobald die erste Baumart, die in einer Zelle vertreten ist, das Alter erreicht hat, um ins Stangenholz zu ge- langen, wird dieser Prozess eingeleitet. In Abhängigkeit von Häufigkeit, Wachstumsgeschwindigkeit und einer Zufallskomponente wird die dominierende Baumart bestimmt. Für jede Baumart wird ein Dominanzfaktor berechnet. Die Wahrscheinlichkeit, dass sich die Fichte als dominierende Art durchsetzt, wird wie folgt hergeleitet:

$$
d_{F I}=0.5+\left[\frac{100}{N_{\text {Alle }}} \times \frac{n_{F 1}+1}{T_{F I}} \times X_{\text {rand }}(1,100)\right]
$$

Dabei ist $\mathrm{n}_{\mathrm{FI}}$ der prozentuale Fichtenanteil der Zelle, $\mathrm{T}_{\mathrm{FI}}$ ist die Verweilzeit der Fichte in Jahren und $\mathrm{X}_{\text {rand }}$ ist eine zufällig bestimmte natürliche Zahl zwischen 1 und 100. Analog werden die Variablen $n$ und T auch für Tanne, Buche und übriges Laubholz bestimmt. $\mathrm{N}_{\text {Alle }}$ wird wie folgt hergeleitet:

$$
N_{\text {Alle }}=\frac{n_{F I}+1}{T_{F I}} \times \frac{n_{T A}+1}{T_{T A}} \times \frac{n_{B U}+1}{T_{B U}} \times \frac{n_{U L}+1}{T_{U L}}
$$

Alle drei Zeitschritte findet eine Pflege (Mischungsregulierung) statt. Bei allen Zellen, die während dieses Zeitschritts vom Jungwald ins Stangenholz wechseln, wird die Mischung nach NaiS bestimmt. Dabei werden in jeder Zelle, die mehr als eine Baumart enthält, auf der Basis der «8-NachbarRegel» Baumarten entfernt, welche gemäss NaiS zu stark vertreten sind. Die «8-Nachbar-Regel» berücksichtigt alle benachbarten Rasterzellen, die direkt horizontal, vertikal oder diagonal erreichbar sind.

Störungen in der Oberschicht werden durch die Prozessgruppe Störungsregime simuliert. In der vorliegenden Version von Rigfor wird nur Windwurf als die häufigste Störung behandelt. In Raum und Zeit gibt es selten grosse und häufig kleine Windwurfereignisse (Oliver \& Larson 1996, Koop \& Hilgen 1987, Pontailler et al 1997). Entsprechend werden die Windwürfe in Rigfor als kleinflächige Störungsereignisse simuliert. Jede Rasterzelle ist im Mittel alle 125 Jahre von einem Sturm betroffen (Hug 2007). Die Auswahl dieser Zellen erfolgt stochastisch. Ein Schaden kann nur eintreten, wenn sich der Wald in der Entwicklungsstufe B I-B III befindet. Die Schaden- 


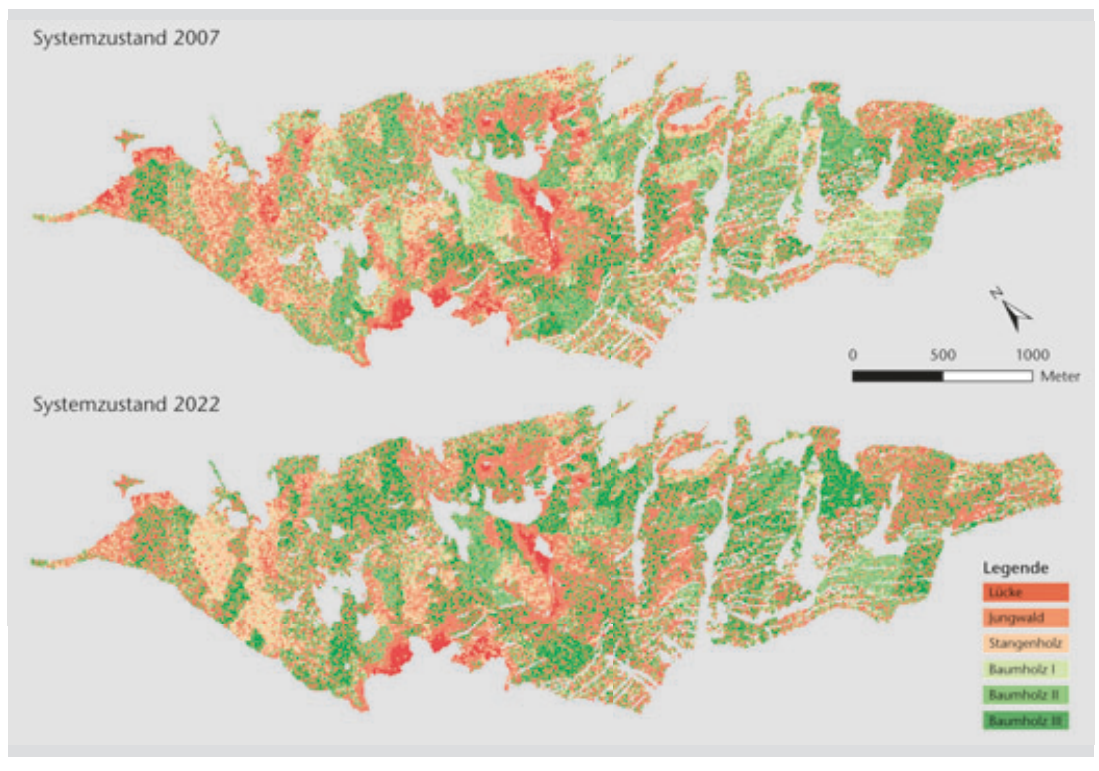

Abb 2 Simulation des Waldzustands in den Jahren 2007 und 2022 mit dem Szenario «aktueller Verbiss» (v1).

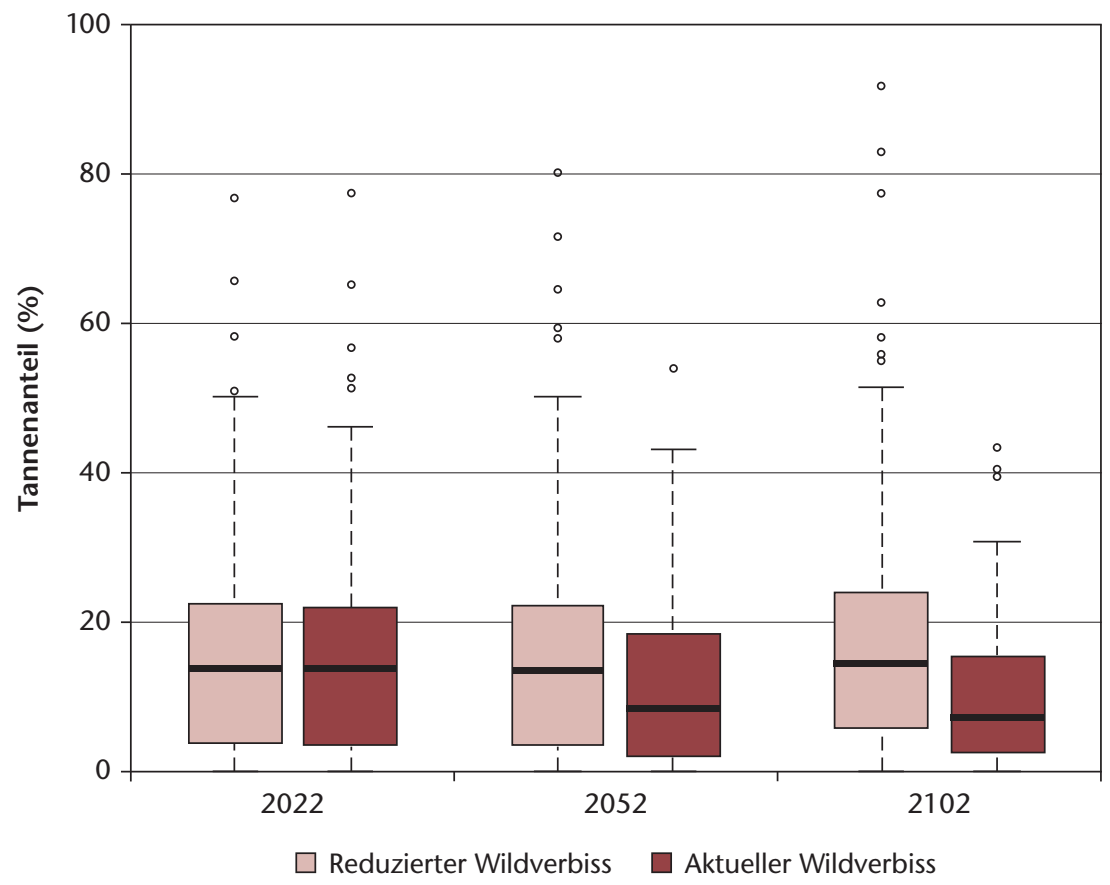

Abb 3 Boxplots der Tannenanteile 2002, 2052 und 2102 im Tannen-Buchen-Wald. Im Vergleich der Szenarien aktueller Verbiss $(v 0)$ und reduzierter Verbiss ( $v 1)$ ergeben sich ab 2052 signifikante Unterschiede (Wilcoxon-Rangsummentest; 2022: p-Wert: 0.69, 2052: P-Wert: 8.6e-11, 2102: P-Wert 2.2e-16).

anfälligkeit ist abhängig von der Baumart (Fichte 71\%, Tanne 66\%, Buche 40\%, übriges Laubholz 30\%; Schütz 2002). Weil an der Rigi eine aktive Borkenkäferbekämpfung stattfindet, werden Borkenkäferschäden in Rigfor nicht berücksichtigt.

\section{Szenarien}

Die Waldentwicklung an der Rigi-Nordlehne wurde in zwei verschiedenen Szenarien während 100 Jahren simuliert. Das Szenario aktueller Verbiss (v1) ist ein sogenanntes Business-as-usual-Szenario, bei dem die Waldentwicklung so weitergeht wie heute. Aufgrund von Wildverbiss ist unter diesem
Szenario der Aufwuchs von Buche und Ahorn im Tannen-Buchen-Wald erschwert, und derjenige der Tanne ist unmöglich. Das Szenario reduzierter Verbiss (v0) unterscheidet sich von v1 darin, dass hier der Wilddruck so stark reduziert wird, dass auch Buche, Ahorn und Tanne aufwachsen können.

\section{Resultate}

\section{Simulierte Waldentwicklung}

Die Wälder an der Rigi-Nordlehne hatten 2007 sowohl einen hohen Anteil an Lücken und Jungwald als auch an Altholz. Einige grössere Lücken gehen bis 2022 in Jungwald über, während andere mangels Samenbäumen Lücken bleiben (Abbildung 2; Abbildungen zu weiteren Zeitschritten und Szenarien vgl. Stadelmann 2008). Selbst in relativ homogenen Beständen, welche sich im Baumholz III befinden, sind Lücken und Jungwaldzellen zu erkennen. Diese konnten auf zwei Arten entstehen: 1) durch das Absterben der Oberschicht oder 2) durch die Herleitung des Anfangszustands, wenn der Deckungsgrad im kartierten Bestand geringer als 100\% war.

\section{Entwicklung der Baumartenanteile mit und ohne Wildverbiss}

Der Wildverbiss ist im Buchenwald an der Rigi-Nordlehne heute tragbar, sodass alle Baumarten aufwachsen können. Folglich sind die Unterschiede zwischen den Szenarien «aktueller Wildverbiss» (v1) und «reduzierter Wildverbiss» (v0) hauptsächlich im Tannen-Buchen-Wald erkennbar. Im Tannen-BuchenWald werden ab 2052 signifikante Unterschiede der Tannenanteile simuliert (Abbildung 3). Unter dem Szenario v1 nimmt der Tannenanteil weiter ab, während er beim Szenario v0 zwischen 2052 und 2102 leicht ansteigt.

Für eine detaillierte Analyse der Baumartenanteile der Szenarien v1 und v0 wurden die sechs Systemzustände zu drei Phasen aggregiert: 1) Lücke und Jungwald, 2) Stangenholz und Baumholz I sowie 3) Baumholz II und III. In allen Phasen wurden zwischen den Szenarien Unterschiede in den Baumartenanteilen festgestellt (Abbildung 4). Im Szenario v1 nimmt der Fichtenanteil zu, der Tannenanteil dagegen sinkt. Einzig im Baumholz II und III werden nur unwesentliche Unterschiede des Tannenanteils simuliert. Für die Buchenanteile zeigt sich ein ähnliches Bild wie bei den Tannenanteilen. Es fällt auf, dass der Buchenanteil in der Oberschicht in beiden Szenarien abnimmt. Die Kategorie übriges Laubholz wird im Szenario v0 etwas häufiger simuliert.

\section{Lücke und Jungwald}

Die Reduktion des Wildverbisses führt über die berücksichtigte Zeitspanne dazu, dass in der Initialphase die Anteile von Tanne, Buche und übrigem 

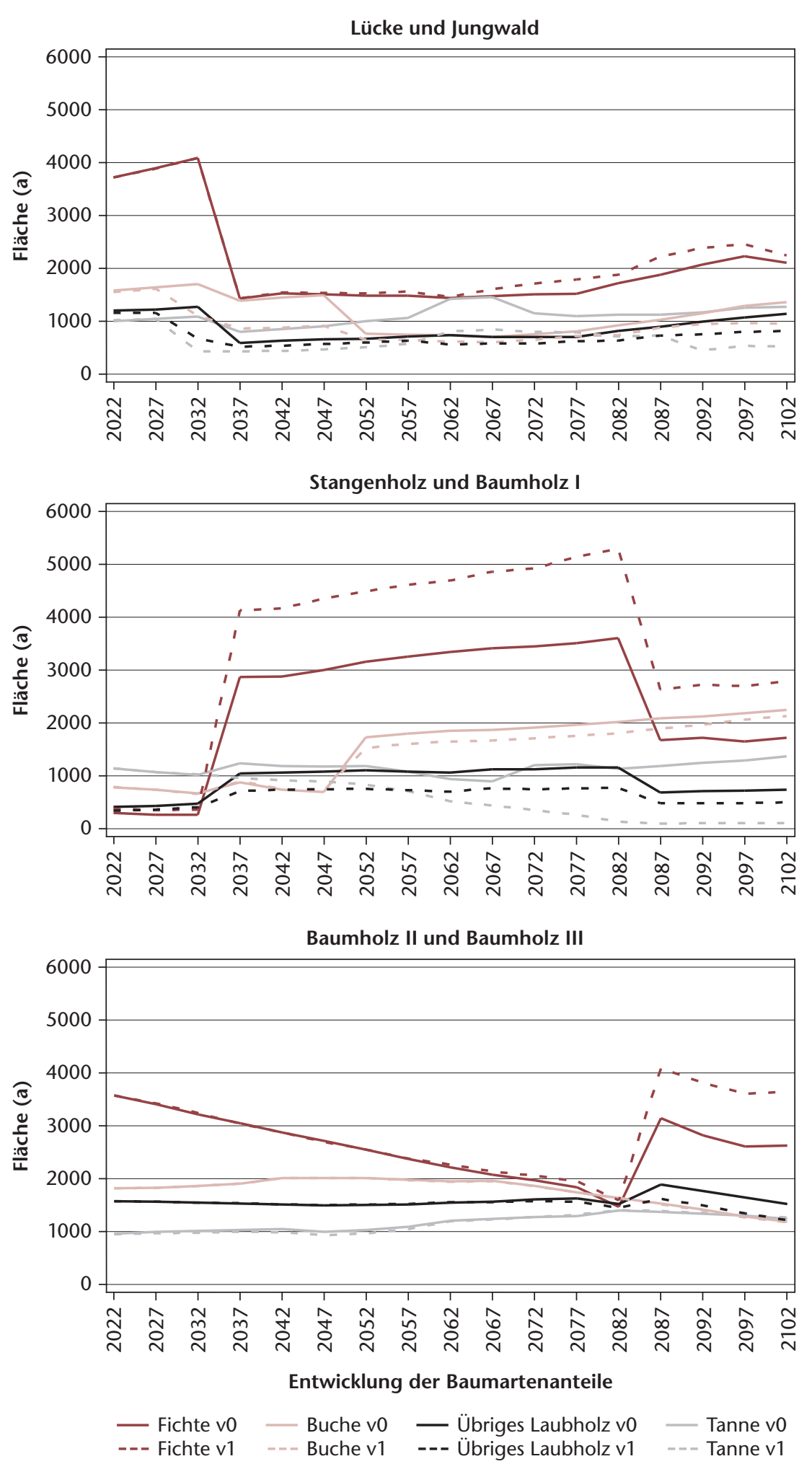

Abb 4 Entwicklung der Baumartenanteile im Tannen-Buchen-Wald in den Szenarien «aktueller Wildverbiss» (v1) und «reduzierter Wildverbiss» (v0). Der Vergleich zeigt, dass beim Szenario «reduzierter Wildverbiss» alle Baumarten ausser der Fichte profitieren.

Laubholz zunehmen, während die Fichtenanteile zurückgehen (Abbildung 4). Am meisten profitiert die Tanne vom reduzierten Wilddruck. Die Fichte bleibt die deutlich häufigste Baumart im Jungwald. Die Fläche mit Fichten steigt bis ins Jahr 2032 kontinuierlich an und wird ähnlich gross wie jene Fläche, welche von den übrigen Baumarten gemeinsam bedeckt ist. Zwischen 2032 und 2037 nimmt die gesamte
Jungwaldfläche stark $a b$, was zu einer Zunahme der Fläche im Stangenholz führt. Besonders deutlich ist dies bei der Fichte erkennbar: Die Jungwaldfläche, auf der die Fichte vertreten ist, sinkt von über 4000 auf unter 2000 Aren ab. Im Übergang zwischen den Jahren 2047 und 2052 wird ein ebenso auffälliger Rückgang der Buchenanteile simuliert.

\section{Stangenholz und Baumholz I}

Bis ins Jahr 2032 werden im Stangenholz und im Baumholz I keine deutlichen Unterschiede zwischen den beiden Szenarien simuliert. Bis zu diesem Zeitpunkt bleibt die Tanne die häufigste Baumart im Stangenholz. Unter dem Szenario aktueller Verbiss (v1) sinkt der Tannenanteil ab 2032 jedoch kontinuierlich ab, bis er im Jahr 2082 auf wenigen Aren stagniert. Fichte und Buche nehmen hingegen unabhängig vom Szenario zu. Bei der Fichte findet diese Zunahme hauptsächlich zwischen 2032 und 2037 statt. Die Buchenanteile steigen zwischen 2047 und 2052 an. Diese Zunahme entspricht der Abnahme der Jungwaldfläche im gleichen Zeitschritt. Die Kategorie übriges Laubholz verzeichnet über den gesamten betrachteten Zeitraum nur eine leichte $\mathrm{Zu}$ nahme. Zwischen 2082 und 2087 wird eine starke Abnahme von Fichte und auch übrigem Laubholz simuliert, weil viele Bäume ins Baumholz II einwachsen.

\section{Baumholz II und III}

Im Baumholz II und III werden bis 2082 keine wesentlichen Unterschiede zwischen den beiden Szenarien simuliert. Während die Tannenanteile bis 2102 leicht ansteigen, ist ab 2067 eine Abnahme der Buche erkennbar. Mit dem Einwachsen von Fichte und übrigem Laubholz zeigen sich ab 2087 die ersten Unterschiede zwischen den Szenarien. Das Szenario «reduzierter Verbiss» (v0) unterscheidet sich dabei vom Szenario «aktueller Verbiss» (v1) dadurch, dass der Fichtenanteil geringer wird. Dafür wird der Anteil des übrigen Laubholzes grösser. Zwischen 2022 und 2082 halbiert sich der Fichtenanteil im Baumholz II und III in etwa.

\section{Diskussion}

\section{Entwicklungsstufen}

Der Wald an der Rigi-Nordlehne wurde jahrzehntelang unternutzt. Dadurch konnte sich ein sehr hoher Holzvorrat entwickeln, der infolge von Sturmereignissen in den Jahren 1987, 1992 und 1999 hauptsächlich in der Buchenwaldstufe reduziert wurde. Im Tannen-Buchen-Wald wurde dagegen im Jahr 2002 immer noch ein grosser Anteil an Baumholz III kartiert. Die Simulationen mit Rigfor zeigen, dass im Tannen-Buchen-Wald der Rigi-Nordlehne die Anteile an Baumholz II und III unabhängig vom Szenario 
während des simulierten Zeitraums konstant bleiben. Der Anteil an Lücken ist zu Beginn der Simulation im Jahr 2002 sehr hoch, da Bestände mit tiefem Deckungsgrad aufgrund der definierten Systemzustände viele Lückenzellen enthalten. Nach einer starken Abnahme bis 2022 bleibt der Lückenanteil bis 2102 praktisch konstant. Die anfangs sehr hohen Anteile an Lücken und Jungwald führen über den simulierten Zeitraum zu deutlichen Schwankungen in den Anteilen des Stangenholzes und des Baumholzes I.

\section{Baumartenverteilung}

Die Verteilung der Baumarten hängt stark vom gewählten Szenario ab. Auswirkungen eines reduzierten Wildverbisses sind zuerst im Jungwald erkennbar, danach im Stangenholz und im Baumholz I, bevor die ersten Unterschiede im Baumholz II und III erkennbar werden. Gerade hier sind die Unterschiede am gravierendsten, da Samenbäume mehrheitlich in diesen Entwicklungsstufen vorkommen. Im Baumholz II und III sind ab 2082 bei Fichte und übrigem Laubholz Unterschiede zwischen den Szenarien v1 und v0 zu beobachten. Die Abnahme der Buche in beiden Szenarien ist bedenklich, da bereits heute nur auf 48\% der Fläche genügend Buchensamenbäume vorhanden sind (Frehner \& Schwitter 2008).

In beiden Szenarien bleiben während der Simulationsperiode genügend Tannensamenbäume bestehen. Im Stangenholz sind jedoch ab 2037 Unterschiede sichtbar. Bei Szenario «reduzierter Wildverbiss» zeichnet sich bis Simulationsende eine leichte Zunahme der Tannenanteile ab. Wenn der Verbiss nicht reduziert wird, geht der Tannenanteil dagegen bis 2082 drastisch zurück, und die Tanne wird langfristig aus dem Bestand verdrängt. Der Rückgang der Tanne ist auf den hohen Wilddruck zurückzuführen, der zu reduziertem Wachstum führt und das Aufwachsen anfälliger Baumarten verhindern kann (Eiberle \& Bucher 1989, Kupferschmid \& Bugmann 2007, Rüegg \& Schwitter 2002, Senn 2000). Bei einem hohen Wilddruck wird sich die Fichte in halboffenen Beständen gegen die Tanne durchsetzen. Unter einem dichten Kronenschirm ist die Verjüngung der Fichte jedoch nicht möglich (Heuze et al 2005a, Heuze et al 2005b), weshalb mit Tanne verjüngt werden muss. An der Rigi werden seit 1990 Beobachtungen gemacht, die belegen, dass Wildverbiss die Hauptursache des fehlenden Tannenaufwuchses ist: Obwohl ein gutes Keimbett vorhanden ist und genügend Sämlinge aufkommen, kann die Tanne dem Äser nicht entwachsen (Frehner \& Schwitter 2008, Hug 2007).

Weil im Tannen-Buchen-Wald an der RigiNordlehne ein hoher Anteil an Baumholz III stockt, ist die Einleitung der Verjüngung dringlich, wenn die Schutzfunktion dieser Bestände langfristig aufrechterhalten werden soll. Für die Verjüngung unter Schirm sind schattentolerante Baumarten wie die
Tanne und die Buche erforderlich. Die Fichte sollte im Tannen-Buchen-Wald einen Anteil von 30\% nicht übersteigen (Frehner et al 2005, Frehner \& Schwitter 2008). Die Tanne dagegen ist sehr erwünscht, da sie mit ihren tiefen Wurzeln weniger sturmanfällig ist (Mayer et al 2005) und nicht vom Buchdrucker befallen wird. Wenn die Tanne weiterhin nicht aufwachsen kann, wird in kleinen Lücken nur Verjüngung aufkommen können, wenn genügend Buchensamenbäume vorhanden sind. Auf etwa der Hälfte der zu verjüngenden Fläche ist dies nicht der Fall.

\section{Verwendete Methode}

Alte Bestände mit einer instabilen Oberschicht sind störungsanfällig. Weil vermehrt mit Störungen zu rechnen ist (North et al 2007), müssen weitere Schäden erwartet werden (Wohlgemuth et al 2008). Die kleinflächigen Störungen, welche in Rigfor simuliert wurden, haben zu einer räumlich heterogenen Verteilung der Systemzustände geführt, d.h., es wurde eine besser werdende vertikale Struktur simuliert. Gegen diese Interpretation spricht, dass der Anteil an Baumholz III in allen Simulationen konstant bleibt. Zwei Faktoren führen dazu, dass die Flächen mit ungenügender Schutzwirkung in der Realität grösser sein dürften, als sie vom Modell simuliert werden. Einerseits könnte der Wald sich bei grossflächigen Störungen, die im Modell nicht simuliert wurden, nur ungenügend erholen, da heute unter Schirm kaum An- und Aufwuchs vorhanden ist. Andererseits ist zu erwarten, dass Konkurrenz durch die Bodenvegetation die Ansamung behindert, wenn der Bestand durch Holzschläge oder Störungen geöffnet wird; diese Konkurrenz wurde ebenfalls nicht modelliert. Dies hat zur Folge, dass die langfristige Schutzwirkung des Waldes abnimmt. Unabhängig vom gewählten Szenario dürften die Flächen mit ungenügender Schutzwirkung bis 2022 ansteigen, da der optimale Zeitpunkt zur Einleitung der Verjüngung in einigen Beständen bereits verpasst wurde. Bei reduziertem Wildverbiss (v0) dürfte die Tanne bis 2050 auf aktuellen Lücken bereits wieder im Stangenholz sein. Anhaltender Wilddruck (v1) verhindert diese Entwicklung jedoch vollständig. Wenn das Szenario v1 eintrifft, muss davon ausgegangen werden, dass der Wald in 50 Jahren seine Schutzfunktion nicht mehr ausreichend erfüllen kann.

Rigfor wurde entwickelt, um die zukünftige Schutzwirkung des Waldes an der Rigi-Nordlehne abzuschätzen. Die Systemzustände in Rigfor wurden aus der Bestandeskarte von 2002 abgeleitet. Dabei wurden bei einem Deckungsgrad unter 100\% Lücken simuliert, um insgesamt die entsprechende Kronendeckung zu erzielen. Dies führte zu einem hohen Lückenanteil im Jahr 2002. In der Simulation ist gut erkennbar, wie sich der hohe Lückenanteil vom Jahr 2002 in den Jungwald und schliesslich ins Stangenholz und ins Baumholz I fortpflanzt. Weil an der 
Rigi-Nordlehne viele Bestände vorratsreich und dunkel sind, können sich in kleinen Lücken aufgrund der Lichtverfügbarkeit nur die Tanne und die Buche entwickeln. In der gegenwärtigen Version von Rigfor wird jedoch die Lichtverfügbarkeit nicht berücksichtigt, weshalb in diesen Lücken oft Fichtenverjüngung simuliert wurde, obwohl für die Fichte zu wenig Licht vorhanden ist. Dadurch wird unter Szenario «aktueller Verbiss» v1 die Entwicklungsgeschwindigkeit von Lücke zu Jungwald überschätzt. Beim Übergang von Jungwald ins Stangenholz findet in Rigfor eine Reduktion auf nur eine Baumart je Zelle (Formeln 1 und 2) statt, wobei eine dominierende Baumart bestimmt wird. Raschwüchsige Baumarten erreichen tendenziell eine zu hohe Dominanz. Folglich wird die Konkurrenzkraft und Entwicklungsfähigkeit der Fichte in den Simulationen überschätzt. Weil sich die Fichte unter Berücksichtigung der Lichtverfügbarkeit weniger stark entwickeln kann, als simuliert wurde, ist zu erwarten, dass kleine Lücken im Tannen-Buchen-Wald nicht einwachsen können. Die tatsächliche Beeinträchtigung der Schutzwirkung im Tannen-Buchen-Wald fällt somit stärker aus, als dies in den Simulationen wiedergegeben wird.

Bisher wurde noch nicht untersucht, welcher finanzielle Schaden auftritt, wenn die Schutzwirkung durch den Wald nicht mehr gewährleistet werden kann. Die Resultate aus der Modellierung mit Rigfor wurden deshalb verwendet, um den Einfluss des Wildverbisses auf den Schutzwald an der RigiNordlehne ökonomisch zu bewerten (Gasser et al 2011, dieses Heft).

\section{Schlussfolgerungen}

Mit dem Modell Rigfor wurde am Beispiel der Rigi-Nordlehne die zukünftige Waldentwicklung unter den zwei Szenarien «aktueller Verbiss» und «reduzierter Verbiss» verglichen. Es zeigte sich, dass bei anhaltendem Wildverbiss die Tanne bis $2082 \mathrm{im}$ Baumholz und im Stangenholz stark reduziert wird. Eine Reduktion der Baumartenvielfalt führt in der Regel auch dazu, dass die Resistenz und die Resilienz des Bestandes gegenüber Störungsereignissen abnehmen (Mayer et al 2005, Wohlgemuth et al 2008). Daher sollte der Wildverbiss so rasch wie möglich so stark reduziert werden, dass die Verjüngung aufwachsen kann (Brang et al 2008, Frehner \& Schwitter 2008, Eiberle \& Bucher 1989). Falls bis in circa zehn Jahren die Verjüngung nicht gewährleistet werden kann, muss damit gerechnet werden, dass der Wald an der Rigi-Nordlehne seine Schutzfunktionen langfristig nicht mehr erfüllen kann, da bereits heute eine Verjüngungslücke von mindestens 20 Jahren besteht.

Eingereicht: 15. August 2010, akzeptiert (mit Review): 12. November 2010

\section{Literatur}

BRANG P, SCHÖNENBERGER W, BACHOFEN H, ZINGG A, WEHRLI A (2004) Schutzwalddynamik unter Störungen und Eingriffen: auf dem Weg zu einer systemischen Sicht. In: Eidgenöss Forsch.anstalt Wald Schnee Landsch, editor. Schutzwald und Naturgefahren. Birmensdorf: Eidgenöss Forsch.anstalt Wald Schnee Landsch, Forum für Wissen. pp. 55-66.

BUGMANN H (1999) Anthropogene Klimaveränderung, Sukzessionsprozesse und forstwirtschaftliche Optionen. Schweiz Z Forstwes 150: 275-287. doi: 10.3188/ szf.1999.0275

DAVIS FW, MORITZ M (2001) Mechanisms of disturbance. In: Simon AL, editor. Encyclopedia of biodiversity. New York: Elsevier. pp. 153-160.

EIBERLE K, BUCHER H (1989) Interdependenzen zwischen dem Verbiss verschiedener Baumarten in einem Plenterwaldgebiet. Z Jagdwissen 35: 235-244.

FREHNER M, SCHWITTER R (2008) Waldgutachten Rigi-Nordlehne 2008. Erfolgskontrolle und Aktualisierung des Waldgutachtens Rigi-Nordlehne 1998. Immensee: Pro Silva Rigi Nord. $28 \mathrm{p}$.

FREHNER M, WASSER B, SCHWITTER R (2005) Nachhaltigkeit und Erfolgskontrolle im Schutzwald. Wegleitung für Pflegemassnahmen in Wäldern mit Schutzfunktion. Bern: Bundesamt Umwelt Wald Landschaft. 564 p.

GASSER N, FREHNER M, ZINGGELER J, OLSCHEWSKI R (2011) Ökonomische Konsequenzen der Verbissprobleme an der RigiNordlehne. Schweiz Z Forstwes 162: 364-371. doi: 10.3188/ szf.2011.0364

HEUZE P, SCHNITZLER A, KLEIN F (2005A) Consequences of increased deer browsing in winter on silver fir and spruce regeneration in the Southern Vosges mountains: Implications for forest management. Ann For Sci 62: 175-181.

HEUZE P, SCHNITZLER A, KLEIN F (2005B) Is browsing the major factor of silver fir decline in the Vosges Mountains of France? For Ecol Manage 217: 219-228.

HUG M (2007) Betriebsplan für das Gebiet Rigi und Rossberg der Schweizerischen Bundesbahn AG (SBB) und des Kantonsforstamtes Schwyz - Gültigkeitsperiode: 2005 bis 2024. Wohlen bei Bern.

KOOP H, HILGEN P (1987) Forest dynamics and regeneration mosaic shifts in unexploited beech (Fagus sylvatica) stands at Fontainebleau (France). For Ecol Manage 20: 135-150. KORPEL S (1995) Die Urwälder der Westkarpaten. Stuttgart: Gustav Fischer. 310 p.

KUPFERSCHMID A, BUGMANN H (2007) Ungulate browsing in winter reduces the growth of Fraxinus and Acer saplings in subsequent unbrowsed years. Plant Ecol 198: 121-134.

MAYER P ET AL (2005) Forest storm damage is more frequent on acidic soils. Ann For Sci 62: 303-311.

NORTH N ET AL (2007) Klimaänderung in der Schweiz. Indikatoren zu Ursachen, Auswirkungen, Massnahmen. Bern: Bundesamt Umwelt, Umwelt-Zustand. $77 \mathrm{p}$.

OLIVER CD, LARSON BC (1996) Forest stand dynamics. New York: Wiley. 520 p.

PONTAILLER JY, FAILLE A, LEMÉE G (1997) Storms drive successional dynamics in natural forests: a case study in Fontainebleau forest (France). For Ecol Manage 98: 1-15.

RÜEGG D, SCHWITTER R (2002) Untersuchungen über die Entwicklung der Verjüngung und des Verbisses im VivianSturmgebiet Pfäfers. Schweiz Z Forstwes 153: 130-139. doi: 10.3188/szf.2002.0130 
SCHÜTZ JP (2002) Die Technik der Waldverjüngung von Wäldern mit Ablösung der Generationen. Zürich: ETH Zürich, Professur Waldbau, Skripten. $144 \mathrm{p}$.

SENN J (2000) Huftiere und Verjüngung im Gebirgswald: eine Geschichte mit vielen Variablen und noch mehr Interaktionen. Schweiz Z Forstwes 151: 99-106. doi: 10.3188/ szf.2000.0099
STADELMANN G (2008) Modellierung der Waldentwicklung an der Rigi-Nordlehne zur Analyse der Schutzwirkung von Wald gegen Murgang. Zürich: ETH Zürich, Professur Waldökologie, Masterarbeiten. 79 p. doi: 10.3929/ethza-005968602

WOHLGEMUTH, T (2008) Effekte des Klimawandels auf Windwurf, Waldbrand und Walddynamik im Schweizer Wald. Schweiz Z Forstwes 159: 336-343. doi: 10.3188/ szf. 2008.0336

\section{Modellierung des Einflusses von Wildver- biss auf die Schutzwaldentwicklung an der Rigi-Nordlehne}

Die Wälder an der Rigi-Nordlehne schützen vor Stein- und Blockschlag, Erosion, Rutschungen, Hochwasser und Murgang. Durch die Bewirtschaftung will man erreichen, dass die Wälder ihre Schutzfunktionen langfristig erfüllen können, indem ein mehrschichtiger Bestandesaufbau mit hoher Störungsresistenz und Resilienz gefördert wird. Zudem wird eine Baumartenmischung gemäss der Wegleitung «Nachhaltigkeit und Erfolgskontrolle im Schutzwald» angestrebt. In der Verjüngung kann heute keine zielgerechte Baumartenmischung erreicht werden, da Wildverbiss im Tannen-Buchen-Wald die Verjüngung behindert.

Um die zukünftige Baumartenmischung voraussagen zu können, wurden mit dem räumlich expliziten Waldentwicklungsmodell Rigfor zwei Szenarien simuliert und miteinander verglichen. Im Szenario «aktueller Wildverbiss» kann die Tanne nicht aufwachsen, und der Aufwuchs von Bergahorn sowie Buche ist erschwert. Im Szenario «reduzierter Wildverbiss» ist hingegen eine zielgerechte Baumartenmischung in der Verjüngung möglich. Die Simulation zeigt, dass die Buchenanteile im Baumholz unabhängig von der Szenarienwahl abnehmen, während bei der Tanne im Baumholz II und III vorerst keine Änderungen erkennbar sind. Bei anhaltendem Wildverbiss werden jedoch ab 2082 praktisch keine Tannen mehr im Stangenholz und im Baumholz I vorhanden sein, was langfristig dazu führen kann, dass die Tanne vollständig verdrängt wird.

Unter einem dunklen Altholzschirm lassen sich die Tanne und die Buche verjüngen, wenn der Wildverbiss auf ein erträgliches Mass reduziert wird. Die Fichte hingegen benötigt bessere Lichtverhältnisse, die an der Rigi-Nordlehne oft nicht vorhanden sind. Um die Schutzwirkung des Waldes an der Rigi-Nordlehne erhalten zu können, müssen die Altholzbestände zielgerecht verjüngt werden. Eine zielgerechte Baumartenmischung in der Verjüngung ist jedoch nur möglich, wenn der Wilddruck reduziert werden kann.

\section{Modélisation de I'influence de l'abroutisse- ment sur le développement de la forêt de protection du versant nord du Rigi}

Les forêts du versant nord du Rigi offrent une protection contre les chutes de pierres, l'érosion, les glissements de terrain, les inondations et les laves torrentielles. Au travers de l'exploitation de ces forêts, dont l'objectif est de créer un peuplement étagé résistant aux perturbations et résiliant, on souhaite pérenniser la fonction protectrice de ces forêts. Pour ce faire, une composition en essences conforme aux directives de la «Gestion durable des forêts de protection» est visée. Dans le rajeunissement aujourd'hui, un mélange d'essences conforme aux exigences ne peut être réalisé en raison de la pression du gibier qui entrave le rajeunissement dans les hêtraies à sapin.

Afin de prédire la future composition en essences, deux scénarios ont été développés et comparés à l'aide du modèle de croissance forestière Rigfor, basé sur des données de terrain explicites. Dans le scénario «pression du gibier actuelle», le développement du sapin blanc est empêché et celui de l'érable sycomore et du hêtre limité. Avec le scénario «pression du gibier réduite», un mélange d'essences conforme à la station est par contre possible. La simulation démontre que la proportion de hêtre au niveau de la futaie va diminuer dans tous les scénarios, alors qu'à court terme, dans la futaie moyenne et la vieille futaie, aucun changement n'est attendu pour le sapin. Avec une pression du gibier au niveau actuel, par contre, il n'y aura quasiment plus de sapin dans le perchis et la jeune futaie à l'horizon 2082, ce qui amènera à long terme à la disparition de cette essence.

Le sapin et le hêtre peuvent être rajeunis sous couvert, si la pression du gibier est réduite à un niveau supportable. L'épicéa, lui, nécessite plus de lumière; une condition difficile à remplir sur le versant nord du Rigi. Pour rajeunir la forêt et maintenir sa fonction protectrice, les vieilles futaies doivent pouvoir être rajeunies selon l'objectif sylvicole. Une composition en essences conforme aux exigences au niveau du rajeunissement n'est possible que si la pression du gibier est réduite. 